\title{
A circumnuclear disk of atomic hydrogen in Centaurus $A$
}

\author{
R. Morganti ${ }^{1,2}$, T. Oosterloo ${ }^{1,2}$, C. Struve ${ }^{1,2}$, and L. Saripalli ${ }^{3,4}$ \\ 1 Netherlands Institute for Radio Astronomy, Postbus 2, 7990 AA, Dwingeloo, The Netherlands \\ e-mail: morganti@astron.nl \\ 2 Kapteyn Astronomical Institute, University of Groningen, Landleven 12, 9747 AD Groningen, The Netherlands \\ 3 Raman Research Institute, CV Raman Avenue, Sadashivanagar, Bangalore 560080, India \\ ${ }^{4}$ CSIRO, Australia Telescope National Facility, PO Box 76, Epping NSW 1710, Australia \\ Received 15 April 2008 / Accepted 5 May 2008
}

ABSTRACT

\begin{abstract}
We present new observations, performed with the Australia Telescope Compact Array, of the Hi absorption in the central regions of Centaurus A. For the first time, absorption is detected against the radio core at velocities blueshifted with respect to the systemic velocity. Moreover, the data show that the nuclear redshifted absorption component is broader than reported before. With these new results, the kinematics of the $\mathrm{HI}$ in the inner regions of Cen $\mathrm{A}$ appears very similar to that observed in emission for the molecular circumnuclear disk. This suggests that the central H I absorption is not, as was previously claimed, evidence of gas infall into the AGN, but instead is due to a cold, circumnuclear disk.
\end{abstract}

Key words. galaxies: active - galaxies: ISM - galaxies: individual: Centaurus A

\section{Introduction}

In the central regions of galaxies with an active galactic nucleus (AGN), gas can play different, sometimes competing roles. This gas is considered to be essential for fuelling the AGN and for turning a dormant black hole into an active one. On the other hand, the gas can also be expelled from these regions as a result of the release of energy from the active nucleus, so that fuelling and star formation are quenched. Moreover, some of the nuclear gas can be arranged in regular structures. Such circumnuclear disks, under certain geometries, can hide the active nucleus from our direct view and can play an important role in explaining the apparent differences between the various types of AGN. The conditions in these central regions are clearly harsh, nevertheless, they are such that not only very dense or highlyionised clouds, but also atomic and molecular gas, can survive.

It has been suggested that neutral hydrogen falling onto the central black hole is a possible mechanism for fuelling an AGN. For many years, the available data seemed to indicate that if $\mathrm{H} \mathrm{I}$ absorption is detected against the core of a radio-load AGN, it is seen at velocities redshifted with respect to the systemic (see, e.g., van Gorkom et al. 1989). Although other factors, such as non-circular motions, can explain redshifted absorption in a single galaxy (see, e.g., García-Burillo et al. 2007), the strong predominance of redshifted absorption has been interpreted as evidence for gas falling into the nucleus. However, it turns out that the relatively narrow observing bands used in the past have led to an incomplete picture (see, e.g., Morganti et al. 2005). Many cases of blueshifted $\mathrm{H}$ I absorption against the radio-core are now known (Vermeulen et al. 2003; Morganti et al. 2005) and as a result, the evidence for nuclear H I absorption to be more often redshifted than blueshifted, and hence for gas infall fuelling the AGN, has disappeared in recent years. A particularly well-known example of possible $\mathrm{H}$ I infall is the detection of redshifted absorption against the nucleus of the closest radio galaxy,
Centaurus $\mathrm{A}^{1}$ (van der Hulst et al. 1983). This case is important because if it were indeed due to gas falling into the AGN, the proximity of Cen A would allow a detailed study of the infall and related processes.

In this Letter, we present new observations of the HI absorption in Cen A that suggest a different interpretation of what causes the HI absorption against the core of this galaxy. The broad observing band used has allowed us to detect, for the first time, blueshifted $\mathrm{H}$ I absorption against the core of Cen A. This obviously complicates the interpretation of the $\mathrm{H}$ I absorption as infall. Below, we discuss the various possibilities and we conclude that the H I absorption is caused by a cold, circumnuclear disk and not by infall into the AGN.

\section{The ATCA data}

Here, we only give a brief summary of the new observations of Cen A, a full description, including results on the largescale gas disk, will be presented in a forthcoming paper (Struve et al., in prep). The observations were done using the Australia Telescope Compact Array (ATCA) in three standard 750-m, 1.5-km, and 6-km configurations, and were carried out on 12, 14, and 20 April 2005 ( $12 \mathrm{~h}$ in each configuration). We chose the selected configurations to obtain good spatial resolution, but at the same time to avoid including too many too short baselines. Given the very strong, very extended continuum emission of Cen A, bandpass calibration is very difficult for short baselines (see below). We used a 16-MHz band with 512 channels, equivalent to a velocity range of $\sim 3000 \mathrm{~km} \mathrm{~s}^{-1}$. This is much broader than used in earlier observations (van der Hulst et al. 1983; Sarma et al. 2002). The centre of the band was set to $V_{\text {hel }}=304 \mathrm{~km} \mathrm{~s}^{-1}$ and the velocity resolution is $13.2 \mathrm{~km} \mathrm{~s}^{-1}$, after Hanning smoothing.

\footnotetext{
1 At the assumed distance of Cen A of $3.5 \mathrm{Mpc}, 1$ arcsec corresponds to $\sim 17 \mathrm{pc}$, or equivalently, 1 arcmin to $1 \mathrm{kpc}$.
} 


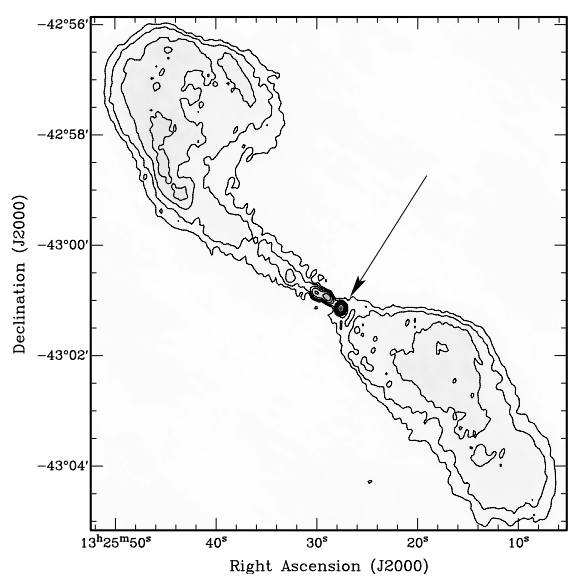

Fig. 1. The continuum image of Cen A obtained from the line-free channels. The absorption discussed here is against the central bright source (indicated by the arrow).

The broad observing band was chosen to ensure that the full width of H I absorption would be sampled if broad signals appeared (as have been seen in other radio galaxies, see Morganti et al. 2005). The source PKS 1934-638 was used as bandpass calibrator. Short observations of about 10 minutes were done every hour on the phase calibrator PKS 1315-46. We carried out the data reduction with the MIRIAD package (Sault et al. 1995).

The extremely strong radio continuum of Cen A ( $>100 \mathrm{Jy}$ on the shortest baseline) makes the bandpass calibration the most delicate part of the calibration. We used the same technique applied by Oosterloo \& Morganti (2005) on earlier observations of Cen A. This involves smoothing the bandpass calibration obtained from PKS 1934-638 with a box-car filter 15 channels wide. This smoothing effectively increases the flux level of PKS 1934-638 to about 60 Jy which is higher than the detected flux on almost all baselines of Cen A. This procedure is allowed because the features in the instrumental bandpass are fairly broad in frequency. This smoothed bandpass correction is applied to the unsmoothed data of Cen A. The resulting spectral dynamic range is better than 1:10000 on the shortest baselines.

We subtracted the continuum using the task UVLIN, making a second order fit to the line-free channels of each visibility record and then subtracting this fit from the spectrum. In order to improve on the phase calibration obtained from PKS 1315-46, we performed frequency independent self-calibration taking advantage of the strong nuclear $\mathrm{H}$ I absorption at some velocities. The final cube was made using the combined datasets. We use the high-resolution cube obtained with uniform weighting and include the $6-\mathrm{km}$ antenna. The lower resolution cubes will be discussed in Struve et al. (in prep.). The restoring beam is $8.1 \times$ 6.8 arcsec with PA $=-12.3^{\circ}$. The rms noise per channel is $\sim 1.3 \mathrm{mJy}_{\text {beam }^{-1}}$. The continuum image obtained from the linefree channels is shown in Fig. 1.

\section{Broad nuclear $\mathrm{HI}$ absorption}

As expected, we detect $\mathrm{HI}$ in emission and, against the strong continuum, in absorption. A full description of these data will be given in Struve et al. (in prep.). We focus on the H I absorption detected against the very central regions.

Both deep H I absorption near the systemic velocity and fainter redshifted absorption against the radio core had been detected before by van der Hulst et al. (1983) and Sarma et al. (2002). Our data show, of course, the deep absorption component near the systemic velocity. However, the new and interesting result is that in our new data, the fainter absorption against the nucleus covers a much larger velocity range compared to previous observations. This larger width of the fainter component is due to both the fact that the redshifted absorption extends to more extreme velocities and to a newly-detected blueshifted component. The absorption spectrum is shown in Fig. 2. The total velocity width is about $400 \mathrm{~km} \mathrm{~s}^{-1}$. The bandwidth used by van der Hulst et al. (1983) was $300 \mathrm{~km} \mathrm{~s}^{-1}$, while that used by Sarma et al. (2002) was only $150 \mathrm{~km} \mathrm{~s}^{-1}$. It is therefore clear that, due to the limited bandwidth used, only part of the redshifted $\mathrm{H}$ I absorption was detected in those observations and that the blueshifted absorption was not detected at all.

The nuclear HI absorption appears asymmetric with respect to the systemic velocity $\left(542 \mathrm{~km} \mathrm{~s}^{-1}\right.$ van Gorkom et al. 1990), covering (heliocentric) velocities from $\sim 400 \mathrm{~km} \mathrm{~s}^{-1}$ (about $-140 \mathrm{~km} \mathrm{~s}^{-1}$ blueshifted compared to systemic) up to $\sim 800 \mathrm{~km} \mathrm{~s}^{-1}$ (i.e., about $+260 \mathrm{~km} \mathrm{~s}^{-1}$ redshifted relative to systemic). The peak absorption has an optical depth of $\tau=0.83$. The column density of the deeper absorption component is $N_{\mathrm{HI}} \sim 2.7 \times 10^{19} T_{\text {spin }} \mathrm{cm}^{-2}$. This column density is in good agreement with what was derived by van der Hulst et al. (1983). One should note that the spin temperature $T_{\text {spin }}$ for H I absorption, occurring close to an AGN, is a very uncertain parameter. Another uncertainty in $N_{\mathrm{HI}}$ is that above we have assumed that the absorption covers the entire underlying continuum source (see also below).

\subsection{A circumnuclear HI disk?}

One of the central questions is of course what structure is causing the faint, broad nuclear HI absorption. The detection of blueshifted absorption obviously complicates the interpretation that it is due to infall. We can exclude that the broad, fainter absorption is produced by gas located at large $(\mathrm{kpc})$ distance from the nucleus. Figure 2 shows that the gas in the large-scale disk is on (almost) circular orbits and that absorption due to the largescale disk against the radio core is near the systemic velocity. Projected on the centre, the large disk shows some spread in velocity (due to non-circular motions) but this spread is much smaller than required to explain the broad central absorption. If some of the absorption would instead occur against the inner continuum jet, the fact that the jet is more or less aligned with the minor axis of the large-scale gas disk means that we would still expect this absorption to be near the systemic velocity. The deep absorption component near the systemic velocity is most likely due to the large-scale gas disk seen in front of the core and jet, but the fainter, broad absorption cannot be caused by it.

One possibility for explaining the nuclear absorption at velocities away from systemic is to assume that the large-scale gas disk is quite thick. In the models of Eckart (1990), the redshifted nuclear absorption components are explained by high-latitude clouds of the large-scale disk at radii of $\sim 500 \mathrm{pc}$. However, such models fail to explain the simultaneous occurrence of bluesifted and redshifted absorption. This means that to reproduce the observed width of the broad, shallow absorption, large radial motions, both inward and outward, would be required to exist in the large-scale disk and such motions are not observed.

An entirely different possibility is that the broad, shallow absorption is caused by a circumnuclear H I disk. If so, one of the prime cases for cold gas falling into an AGN would disappear.

The presence of a cold circumnuclear disk with a size of roughly 100 parsec has been suggested by a number of studies (see, e.g., Israel et al. 1990, and references therein). The central 

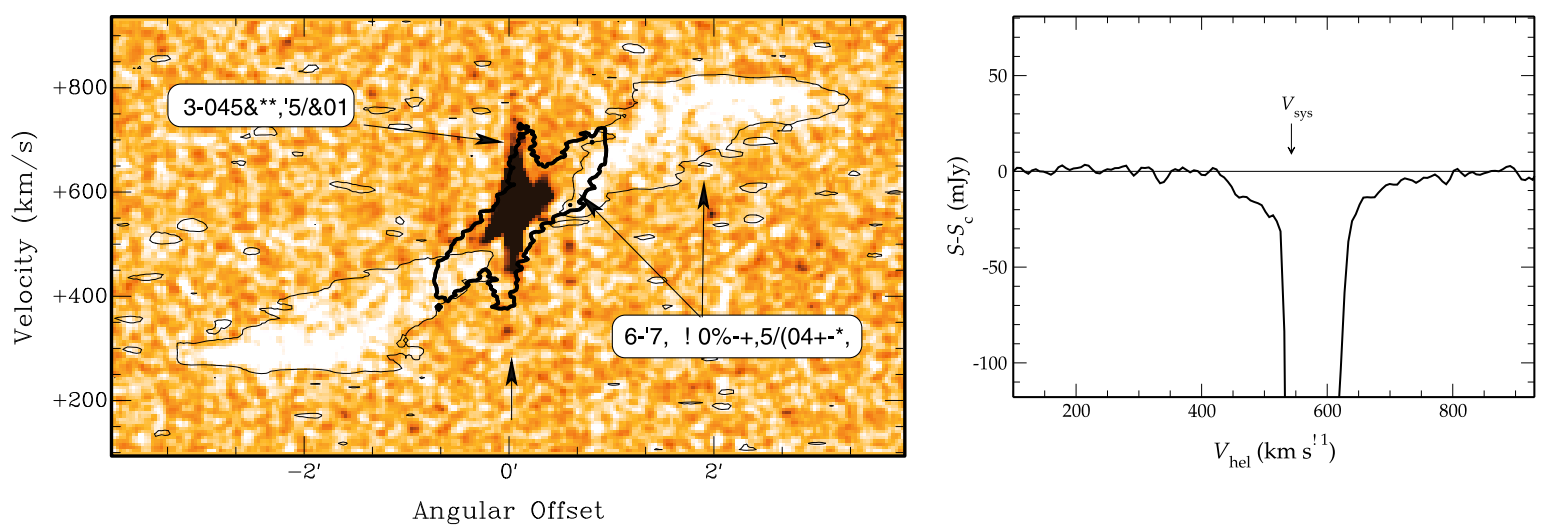

Fig. 2. Left: position-velocity plot of the H I (grey-scale and thin contours) and superimposed the CO emission Liszt (thick contours; from 2001), taken along position angle $139^{\circ}$. The gray-scale represents the high-resolution H I data (beam 8 arcsec) while the thin contour is from the same dataset smoothed to 15 arcsec. Note that the $\mathrm{CO}$ observations do not extend beyond a radius of about 1 arcmin. Right: the absorption profile against the central radio core (indicated by the arrow in the left panel) showing the blue- and redshifted wings of the absorption profile.

regions of Cen A are highly obscured in the optical, but they have been studied in detail using observations at longer wavelengths. Ionised and molecular gas was observed in the $K$-band with both long-slit (Marconi et al. 2001) and integral-field spectroscopy (Krajnović et al. 2007; Neumayer et al. 2008). The most recent observations (Neumayer et al. 2008) show that in the central few arcseconds (i.e. tens of $\mathrm{pc}$ ) very different morphologies are found between the high- and low-ionisation lines. The highly-ionised gas shows a strong kinematical influence of the jet. On the other hand, the molecular gas $\left(\mathrm{H}_{2}\right)$ appears to be in a regularly rotating disk, influenced only by gravity. The velocity channel maps (Fig. 8 in Neumayer et al. 2008) show the kinematics of this inner molecular disk, with velocities ranging from $\sim-230 \mathrm{~km} \mathrm{~s}^{-1}$ to $\sim 230 \mathrm{~km} \mathrm{~s}^{-1}$ compared to systemic. Part of this velocity range is due to the velocity dispersion of the gas, whose origin is not known, and the rest is due to rotation. The $\mathrm{H}_{2}$ kinematics are best modelled by a tilted-ring model of a warped gas disk that, in the inner part, appears quite face on $\left(34^{\circ}\right)$ and with position angle of the kinematic major axis of $\sim 155^{\circ}$.

Other evidence for a circumnuclear molecular disk comes from CO observations (Eckart 1990; Israel et al. 1990; Rydbeck et al. 1993). In particular, CO $J=3-2$ observations (Liszt 2001) have been explained by a disk of radius $168 \mathrm{pc}$ seen nearly edgeon (in position angle $138.5^{\circ}$ on the sky, therefore perpendicular to the base of the radio jet). In these observations, which have a resolution of 14 arcsec, this disk is barely resolved. For both the $\mathrm{CO}$ and the $\mathrm{H}_{2}$ data the kinematic modelling is somewhat uncertain. Nevertheless, the difference in the inclination derived for the inner $\mathrm{H}_{2}$ disks and the somewhat larger $\mathrm{CO}$ structure suggests that it is heavily warped at all spatial scales.

In an attempt to see whether the broad H I absorption could be related to this circumnuclear disk, we have compared the CO $J=3-2$ data (kindly provided by $\mathrm{H}$. Liszt) and the $\mathrm{H}$ I data cubes. Figure 2 shows the position-velocity plot obtained along a line (position angle $139^{\circ}$ ) through the nucleus of Cen A. The gray-scale and the thin contours represent the H I data, while the $\mathrm{CO}$ data is given by the thick contours. Note that the field of view of the CO observations was only $2 \times 2$ arcmin so the CO information does not extend beyond 1 arcmin radius. The $\mathrm{CO}$ data clearly show two kinematical components: a large component with a relatively shallow velocity gradient and a smaller, inner component with a steep velocity gradient. The large $\mathrm{CO}$ component corresponds to the large-scale gas disk associated with the dustlane, while the inner component is the circumnuclear disk modelled by Liszt (2001). The comparison with the H I clearly suggests that both the outer (in emission, and part in absorption) and the inner component (in absorption) are seen also in HI. Figure 2 illustrates the point made earlier that the kinematics of the large disk is clearly such that it cannot explain the large velocity range seen in the very centre.

For the inner part, Fig. 2 shows that the broad, inner H I absorption corresponds very well with the fast rotating, inner $\mathrm{CO}$ disk. In particular, the redshifted H I absorption covers the same velocity range as the $\mathrm{CO}$, suggesting that it is not associated with infalling gas. It is also clear that the $\mathrm{HI}$ and $\mathrm{CO}$ show differences: the extent of the $\mathrm{CO}$, both spatially and in the blueshifted velocities, is larger than of the inner H I. Such differences can be expected given that the characteristics of the inner $\mathrm{HI}$ absorption is set by the size of the background continuum emission, not by the H I structure itself. With the spatial resolution of our H I data ( $8 \operatorname{arcsec})$, the inner H I absorption is unresolved while the total extent of the $\mathrm{CO}$ disk is about 20 arcsec. If the background continuum against which we see the H I absorption is smaller than the circumnuclear CO disk (see below), this would explain the apparent difference in size and velocity range.

\subsection{The nature of the absorbed continuum}

As already eluded to above, for the interpretation of the H I absorption, it is important to understand what the continuum background could be. It turns out that this is not easy to unravel. It is natural to assume that the strongest absorption is against the very inner radio core. However, this is unlikely to be the case. The radio continuum emission on the sub-kpc scale has been studied with high-frequency Very Large Array (about 0.5 arcsec resolution at $8.4 \mathrm{GHz}$, Hardcastle et al. 2003) as well as VLBI observations at frequencies $2.3 \mathrm{GHz}$ and higher, with very high spatial resolution (a few milliarcsec resolution at $2.3 \mathrm{GHz}$; Jones et al. 1996; Tingay et al. 1998). The highest frequency data show that, on parsec and sub-parsec scales, the radio continuum in the central region is dominated by a core-jet structure. However, the VLBI data also show that the core has a stronglyinverted spectrum and that it is actually not visible at $2.3 \mathrm{GHz}$. This suggests that the very innermost part of the radio source (up to $\sim 0.4-0.8 \mathrm{pc}$ ) is seen through a disk or torus of ionised gas which is opaque at lower frequencies due to free-free absorption (Tingay \& Murphy 2001). The above means that the radio core 
is unlikely to be detected also at $1.4 \mathrm{GHz}$, and it is not the core itself against which the $21-\mathrm{cm}$ absorption is occurring.

Given that, at $1.4 \mathrm{GHz}$, the radio core is not seen, the radio continuum on the milli-arcsec scale appears concentrated in the jet and counter-jet. Given the circumnuclear disk structure discussed above, with a PA close to perpendicular to the jet axis, the fact that the main jet is coming toward us means that it is likely to be in front of the circumnuclear disk and that it cannot cause the absorption. One possibility would be then that the HI absorption is against the counter-jet. However, this can be excluded, given the small opening angle of the jet, as most of the absorption would be seen at velocities close to systemic.

An interesting clue is that extra flux (at least $50 \mathrm{mJy}$ ) is clearly present on the short baselines in the 1.4-GHz VLBI experiments (Tingay priv. comm.), indicating the presence of largescale structures that are not evident in the images. This structure would be still well inside the $1 \operatorname{arcsec}(\sim 18 \mathrm{pc})$ imaged at the highest VLA resolution (for $21 \mathrm{~cm}$ ). It is difficult to quantify the amount of flux on larger scales from a comparison of VLBI data with lower-resolution data due to the variability of the nuclear regions (Romero et al. 1997; Abraham et al. 2007) because simultaneous observations are needed. It is therefore unclear whether this extra component can cause the absorption.

If, however, this were the case, we would have a situation similar to the one suggested to be the case for Cygnus A (Conway \& Blanco 1995; Conway 1999). In this source, the H I opacity peaks off the jet, on the counter-jet side, with maximum opacities occurring north and south of the jet axis. The counter-jet of Cyg A is too narrow to explain the width of the absorption. Therefore, Conway (1999) suggested that a more diffuse component could cause the absorption. This component could be due to thermally-emitting ionised gas evaporated from the inner edge of a torus or disk. Other evidence of H I absorption against a diffuse component has been found in Mrk 231 (Carilli et al. 1998). In this object, they identify the H I and the radio continuum disk as the inner part of the molecular disk seen on the larger scale.

If the scenario described above is correct, we can make some remarks about the conditions of the $\mathrm{H}$ I material and whether the location of the H I and of the molecular gas are what is expected from the physical models of the circumnuclear disks/tori. In this case, the column density of the H I is going to be much larger than estimated above, because the absorption will likely cover only a small fraction of the sub-arcsec continuum. For example, if the absorption is only against the counterjet-side, and assuming the jet/counterjet ratio from Jones et al. (1996) of 4-8, the column density is at least a factor four higher. This factor would be even higher in the case of the absorption against the more diffuse component. In addition, in a circumnuclear structure, $T_{\text {spin }}$ is likely to be a few $\times 10^{3} \mathrm{~K}$, as the $\mathrm{HI}$ is affected by the central AGN (Maloney et al. 1996). Therefore the column density could easily be as high as $5 \times 10^{23} \mathrm{~cm}^{-2}$. Interestingly, this is a similar column density as derived from the hard X-ray spectrum of Cen A, which is fitted with an heavily absorbed power-law model with a column density of $\sim 10^{23} \mathrm{~cm}^{-2}$ (Evans et al. 2004).

Physical models of circumnuclear disks/tori have been investigated by Maloney et al. (1994, 1996, and refs. therein). They identify an effective ionisation parameter that determines whether, given the distance from the nucleus, the material is atomic or molecular. This effective ionisation parameter is $\xi_{\text {eff }}=L_{X} N_{22}^{-0.9} n^{-1} r^{-2}$, where $L_{\mathrm{X}}$ is the luminosity of $>2 \mathrm{keV} \mathrm{X}$ rays; $n$ is the gas density; $r$ is the distance to the nucleus; and $N_{22}$ is the total column density in units of $10^{22} \mathrm{~cm}^{-2}$. For $\xi_{\text {eff }}>10^{-3}$, the gas will be largely atomic, otherwise mainly molecular. For Cen A, the X-ray luminosity is $L_{\mathrm{X}}=5 \times 10^{41} \mathrm{erg} / \mathrm{s}$ (Evans et al. 2004) and the $X$-ray column density is $10^{23} \mathrm{~cm}^{-2}$. A rough estimate of the density can be derived from the H I column density if we assume that the region of absorption is comparable to the region with free-free absorption (that covers the innermost $\sim 0.4-0.8 \mathrm{pc}$ ). This gives a density of $1-2 \times 10^{5} \mathrm{~cm}^{-3}$. These values give a radius of $1-3 \mathrm{pc}$ for $\xi_{\mathrm{eff}}=10^{-3}$. Thus, we may hypothesise that the central region of Cen $\mathrm{A}$ hosts a gaseous disk composed of multiple phases where the inner part (but outside the fully ionised region) is mainly atomic and beyond that molecular.

\section{Conclusions}

Using new, broadband, H I observations of Cen A we have shown that the nuclear absorption is broader than previously known and that it has a blueshifted component. Using various arguments, but in particular the comparison with the molecular gas data, we have shown that the HI absorption is likely to be caused by a cold, circumnuclear disk and that it does not constitute direct evidence of gas infall into the AGN.

Sensitive VLBI observations are now needed to further explore the characteristics of the nuclear H I as well as the picture proposed in this paper and, in general, to learn more about the central regions of this fascinating, nearby AGN.

Acknowledgements. We are most grateful to Harvey Liszt for providing us the $\mathrm{CO}$ cube that has been crucial in this work. Based on observations with the Australia Telescope Compact Array (ATCA), which is operated by the CSIRO Australia Telescope National Facility. This research was supported by the EU Framework 6 Marie Curie Early Stage Training programme under contract number MEST-CT-2005-19669 ESTRELA.

\section{References}

Abraham, Z., Barres de Almeida, U., Dominici, T. P., \& Caproni, A. 2007, MNRAS, 375, 171

Carilli, C. L., Wrobel, J. M., \& Ulvestad, J. S. 1998, AJ, 115, 928

Conway, J. E. 1999, in Highly Redshifted Radio Lines, ed. C. L. Carilli, S. Radford, K. Menten, \& G. Langston, ASP Conf. Ser., 156, 259 Conway, J. E., \& Blanco, P. R. 1995, ApJ, 449L, 131

Eckart, A., Cameron, M., Rothermel, H., et al. 1990, ApJ, 363, 451 Evans, D., Kraft, R., Worrall, D., et al. 2004, ApJ, 612, 786 García-Burillo, S., Combes, F., Neri, R., et al. 2007, A\&A, 468, L71 Israel, F. P., van Dishhoeck, E. F., Baas, F., et al. 1990, A\&A, 227, 342 Jones, D. L., Tingay, S. J., Murphy, D. W., et al. 1996, ApJ, 466, L63 Krajnović, D., Sharp, R., \& Thatte, N. 2007, MNRAS, 374, 385 Liszt, H. S. 2001, A\&A, 371, 865

Maloney, P., Hollenbach, D., \& Tielens, A. 1996, ApJ, 466, 561 Marconi, A., Capetti, A., Axon, D. J., et al. 2001, ApJ, 549, 915 Morganti, R., Tadhunter, C. N., \& Oosterloo, T. 2005, A\&A, 444, L9 Neumayer, N., et al. 2008, ApJ, in press [arXiv:0709.1877]

Oosterloo, T. A., \& Morganti, R. 2005, A\&A, 429, 469

Quillen, A. C., Brookes, M. H., Keene, J., et al. 2006, ApJ, 645, 1092 Rydbeck, G., Wiklind, T., Cameron, M., et al. 1993, A\&A, 270, L13 Romero, G. E., Benaglia, P., \& Combi, J. A. 1997, A\&AS, 124, 307 Sarma, A. P., Troland, T. H., \& Rupen, M. P. 2002, ApJ, 564, 696

Sault, R. J., Teuben, P. J., \& Wright, M. C. H. 1995, in Astronomical Data Analysis Software and Systems IV, ed. R. Shaw, H. E. Payne, \& J. J. E. Haynes, ASP Conf. Ser., 77, 433

Tingay, S. J., \& Murphy, D. W. 2001, ApJ, 546, 210

Tingay, S. J., Jauncey, D. L., Reynolds, J. E., et al. 1998, ApJ, 115, 960 van der Hulst, J. M., Golish, W. F., \& Haschick, A. D. 1983, ApJ, 264, L37 van Gorkom, J. H., Knapp, G. R., Ekers, R. D., et al. 1989, AJ, 97, 708 van Gorkom, J. H., van der Hulst, J. M., Haschick, A. D., \& Tubbs, A. D. 1990, AJ, 99, 1781

Vermeulen, R. C., Pihlström, Y. M., Tschager, W., et al. 2003, A\&A, 404, 861 